\title{
Spontaneous intercalated corneal epithelial folds in thyroid eye disease: a case report
}

Christine CY Lam, ${ }^{l}$ Kelvin KL Chong, ${ }^{2,3}$ MBChB(Hon), FCOphthHK

${ }^{1}$ LKS Faculty of Medicine, The University of Hong Kong, Hong Kong

${ }^{2}$ Department of Ophthalmology and Visual Sciences, Prince of Wales Hospital, Hong Kong

${ }^{3}$ Department of Ophthalmology and Visual Sciences, The Chinese University of Hong Kong, Hong Kong

Correspondence and reprint requests:

Dr Kelvin KL Chong, Department of Ophthalmology and Visual Sciences, The Chinese University of Hong Kong, Hong Kong. Email: chongkamlung@cuhk.edu.hk

\section{Abstract}

We observed an under-recognized, ethnic-related sign in thyroid eye disease in a 41-year-old Chinese woman. Spontaneous corneal epithelial folds form after fluorescein staining as a result of tear film disruption related to orbital tension and upper lid pressure on the cornea. They occur in anatomically predisposed eyes similar to acquired lower lid epiblepharon. Like their chorioretinal counterparts, presence of corneal epithelial folds prompt for further workup, even in East Asian patients whose thyroid eye changes may appear less impressive.

Key words: Asian continental ancestry group; Epithelium,

corneal; Graves ophthalmopathy

\section{Case presentation}

In June 2018, a non-smoking 41-year-old Chinese woman presented with a 3-month history of worsening periocular swelling and tightness. She had a 4-year history of stable Graves disease after radioactive iodide treatment, and she was taking $50 \mathrm{mg}$ thyroxine supplement. On examination, visual acuity of each eye was 20/30, with normal Ishihara and pupillary responses and visual field on confrontation. Intraocular pressure was $13 \mathrm{~mm} \mathrm{Hg}$ and $11 \mathrm{~mm} \mathrm{Hg}$ at primary gaze and $17 \mathrm{~mm} \mathrm{Hg}$ and $19 \mathrm{~mm} \mathrm{Hg}$ on upgaze for right and left eye, respectively. Spontaneous, intercalated corneal epithelial (CE) folds were evident only after fluorescein eyedrop staining and under cobalt-blue lights (Figure 1) before applanation tonometry. She had bilateral upper lid puffiness, retraction, and increased resistance to retropulsion. Despite having mild exophthalmos of $18 \mathrm{~mm}$ bilaterally on Hertel exophthalmometry, normal eye movement, and a low clinical activity score of 1 on eyelid swelling, she was referred for further oculoplastic evaluation that showed no chorioretinal fold or optic disc swelling. The patient's anterior chamber depth, intraocular pressures, anterior segment optical coherence tomography, and corneal topographies (Figure 3) were all normal.

Magnetic resonance imaging demonstrated minimally enlarged or inflamed extraocular muscles with predominant fat expansion (Figure 2). Combined intravenous pulse methylprednisolone with orbital radiotherapy was proposed as compassionate treatment for progressive and symptomatic thyroid eye disease despite a low clinical activity score, which was well tolerated.

At the 3-month follow-up, periocular swelling and exophthalmos improved, but CE folds persisted. Patient was offered options of staged rehabilitative orbital decompression, upper lid recession, and blepharoplasty for residual deformities. At the 21-month follow-up, CE folds were static.

\section{Discussion}

To the best of our knowledge, there has been only one case report describing corneal stromal (deep) striae (folds) persisting after endoscopic decompression for optic neuropathies in an Asian woman with thyroid eye disease. ${ }^{1}$ These corneal stromal striae were evidence of raised orbital 

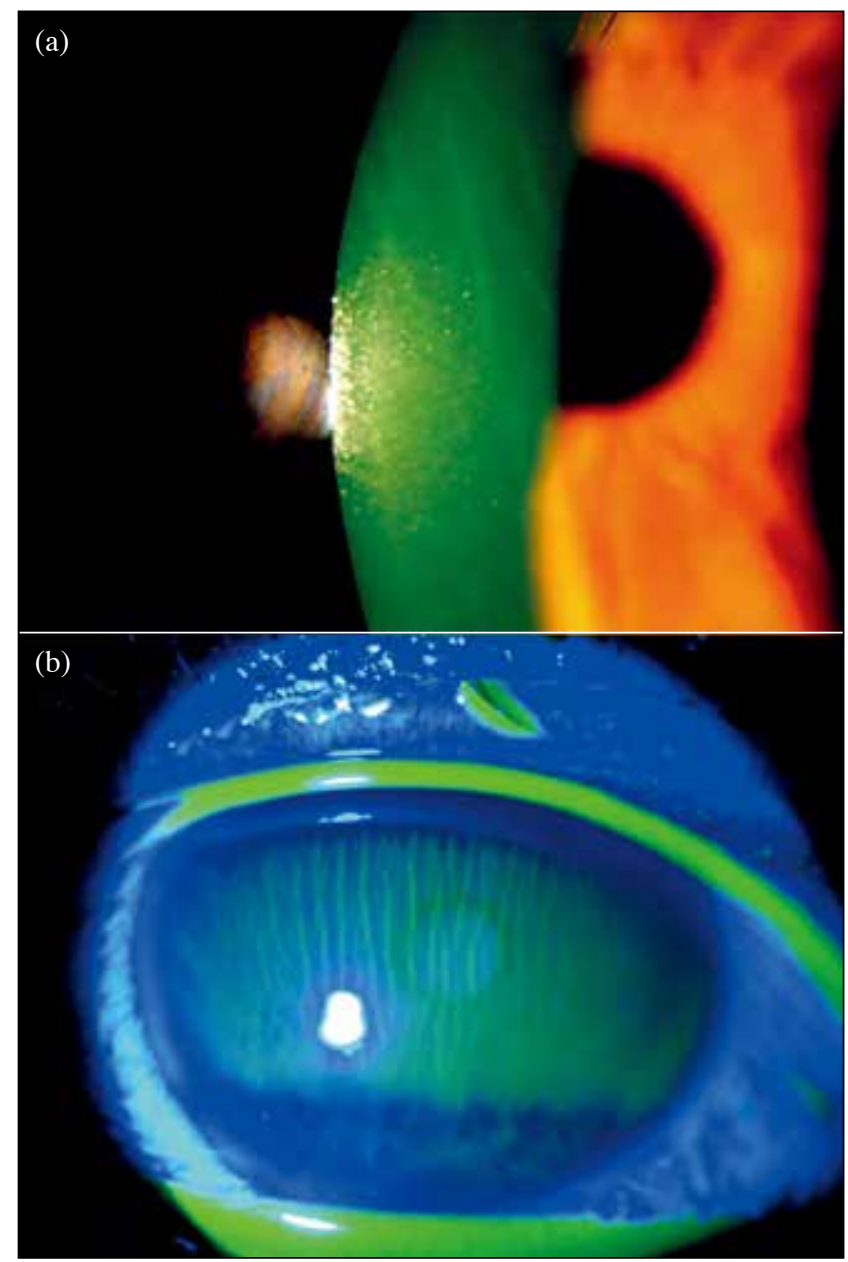

Figure 1. Corneal epithelial folds in an eye with formed anterior chamber and clear cornea visible under (a) fluorescein staining and (b) cobalt-blue lights

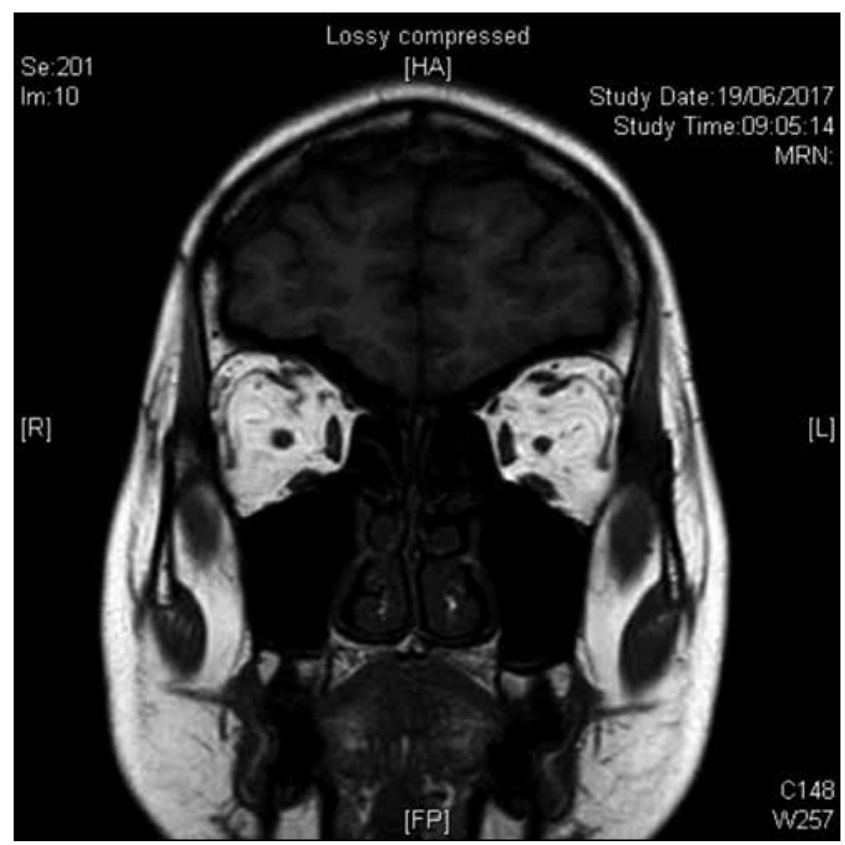

Figure 2. Magnetic resonance imaging showing normal-sized extraocular muscles with fat expansion

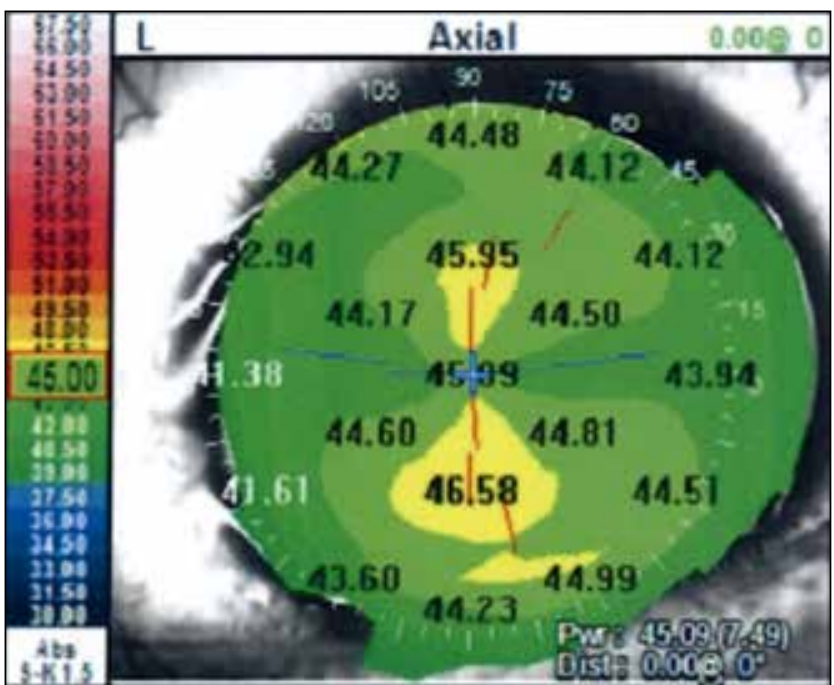

Figure 3. Unremarkable corneal topography

pressure and were resolved upon further three-wall orbital decompression and radiotherapy. ${ }^{1}$ In our patient, the CE folds were superficially located as alternating bands of negative staining. They were present only after fluorescein staining and indicated tear film disruption over the elevated, optically disruptive epithelial microfolds. The patient's anterior chamber depth, intraocular pressures, anterior segment optical coherence tomography, and corneal topographies were all normal.

Folds form at the front (cornea) or back (chorioretina) of the eye when tissue layers buckle back as vertical lines. Chorioretinal folds are another sign indicating raised orbital pressure in thyroid eye disease. ${ }^{2}$ We propose that CE folds or stromal striae are more prevalent among the anatomically predisposed eyes, similar to acquired lower lid epiblepharon in thyroid eye disease..$^{3-5}$ In East Asians whose upper lid creases are low or absent, progressive fat expansion beneath a tight orbital septum exerts pressure on the most superficial (ie epithelial) layer of the cornea leading to formation of $\mathrm{CE}$ folds.

In our patient, $\mathrm{CE}$ folds did not interfere with visual acuity, analogous to microstriae after LASIK, although contrast sensitivity, higher order aberration or Maddox rod effect was not evaluated. ${ }^{6}$ Similar to chorioretinal folds, there is no treatment guideline for $\mathrm{CE}$ folds per se in thyroid eye disease. Soft tissue signs improved although $\mathrm{CE}$ folds persisted after combined pulse methylprednisolone and radiotherapy. For patients with no demonstratable visual consequence, continuing management of any associated ocular surface problem (eg dry eye) is an acceptable option. In patients with symptomatic periocular deformities (including upper lid swelling, retraction, and exophthalmos), structural changes after orbital decompression, upper lid recession, and blepharoplasties may lead to resolution of CE folds. Persistent chorioretinal folds, attributed to scleral remodeling, was reported despite complete removal of retrobulbar tumor? 


\section{Conclusion}

The presence of $\mathrm{CE}$ or chorioretinal folds in patients with thyroid eye disease may prompt further oculoplastic evaluation or radiological workup, especially in East Asian patients whose periorbital deformities or disease activities are often minimal or subtle.

\section{Author contributions}

Concept or design: All authors

Acquisition of data: All authors

Analysis or interpretation of data: All authors

Drafting of the article: All authors

Critical revision for important intellectual content: All authors

All authors had full access to the data, contributed to the study, approved the final version for publication, and take responsibility for its accuracy and integrity.

\section{Conflicts of interest}

As an editor of the Journal, KKL Chong was not involved in the peer review process for this article. Other authors have disclosed no conflicts of interest.

\section{Funding/support}

This research received no specific grant from any funding agency in the public, commercial, or not-for-profit sectors.

\section{Ethics approval}

The patient was treated in accordance with the Declaration of Helsinki. The patient provided written informed consent for treatments and procedures and for publication.

\section{References}

1. Kashani S, Papadopoulos R, Olver J. Corneal striae in thyroid eye disease. Eye (Lond) 2007;21:869-70. Crossref

2. Vahdani $K$, Rose GE. Chorioretinal folds in thyroid eye disease. Ophthalmology 2019;126:1106. Crossref

3. Chang EL, Hayes J, Hatton M, Rubin PA. Acquired lower eyelid epiblepharon in patients with thyroid eye disease. Ophthalmic Plast Reconstr Surg 2005;21:192-6. Crossref

4. Park SW, Khwarg SI, Kim N, Lee MJ, Choung HK. Acquired lower eyelid epiblepharon in thyroid-associated ophthalmopathy of Koreans. Ophthalmology 2012;119:390-

5. Crossref

5. Zhao J, Hodgson NM, Chang JR, Campbell AA, McCulley TJ. Thyroid eye disease-related epiblepharon: a comparative case study. Asia Pac J Ophthalmol (Phila) 2020;9:447. Crossref

6. Choi CJ, Melki SA. Maddox rod effect to confirm the visual significance of laser in situ keratomileusis flap striae. J Cataract Refract Surg 2011;37:1748-50. Crossref

7. Jacobsen AG, Toft PB, Prause JU, Vorum H, Hargitai J. Long term follow-up of persistent choroidal folds and hyperopic shift after complete removal of a retrobulbar mass. BMC Res Notes 2015;8:678. Crossref 